\title{
Comparison of Different Binocular I Pad Games with Patching for Treatment of Amblyopia in Children: Pilot Study
}

\author{
Kholoud Ahmed Bokhary ${ }^{1}$, , Latifah Saud Aldohayan ${ }^{1}$, Fahmy Rania Medhat ${ }^{1,2}$, \\ Abdullah Ghazi Alotaibi ${ }^{3}$ \\ ${ }^{1}$ Department of Optometry and Vision Science, Collage of Applied Medical Science, King Saud University, Riyadh, Kingdom of Saudi \\ Arabia \\ ${ }^{2}$ Department of Ophthalmology, Faculty of Medicine, Cairo University, Giza, Egypt \\ ${ }^{3}$ Department of Ophthalmology, College of Medicine, King Saud University, Riyadh, Kingdom of Saudi Arabia
}

Email address:

kbokhary@ksu.edu.sa (K. A. Bokhary)

*Corresponding author

\section{To cite this article:}

Kholoud Ahmed Bokhary, Latifah Saud Aldohayan, Fahmy Rania Medhat, Abdullah Ghazi Alotaibi. Comparison of Different Binocular I Pad Games with Patching for Treatment of Amblyopia in Children: Pilot Study. International Journal of Ophthalmology \& Visual Science. Vol. 5, No. 1, 2020, pp. 31-34. doi: 10.11648/j.ijovs.20200501.16

Received: December 22, 2019; Accepted: December 30, 2019; Published: January 31, 2020

\begin{abstract}
Purpose- The aim of the study is to compare visual functions of children with uncorrected amblyopia treated with different binocular I Pad games and/or patching. Methods- Children with untreated amblyopia were enrolled in this pilot study. Children were divided into three groups: children treated with patching only, children attributed to play binocular snake I Pad game with patching, children attributed to play binocular blocks I Pad game with patching. Visual acuity VA, refraction, stereo acuity test, and ocular alignment tests were performed for all participants in the first visit and 4 weeks after starting treatment. Visual functions were compared between both visits. Results- A total of 18 children with untreated amblyopia participated in this study. Children were divided into three groups: Group $1=8$ children treated with patching only, Group $2=5$ children attributed to play binocular snake I Pad game with patching, and Group $3=5$ children attributed to play binocular blocks I Pad game with patching. There was no significant difference $(\mathrm{P}>0.05)$ in gender and age between the three groups. Children showed good compliance to all patching treatment and I pad games groups. After 4 weeks, Visual acuity was improved in all amblyopic children in the form of one line improvement in patching group, two lines with binocular snake I Pad game and three lines with binocular blocks I Pad game. Moreover, stereoacuity was improved in all groups with greatest advance in blocks games group followed by snake group then patching group. Conclusion- This study demonstrated great effectiveness of both snake, and blocks binocular I Pad games on visual functions improvement for treatment of amblyopia. These games can contribute with patching in achieving better VA and stereoacuity enhancement in a shorter duration than patching alone.
\end{abstract}

Keywords: Amblyopia, I Pad Games, Patching, Stereoacuity, Visual Acuity

\section{Introduction}

Amblyopia (Lazy eye) was defined as "decreased vision in one or both eyes as a result of insufficient stimulation of the development of the visual system" [1]. It is clinically a change in the visual acuity between the two eyes that is equal or more to $0.2 \log$ MAR.

The prevalence of amblyopia is varying worldwide. For example, in Saudi Arabia, the prevalence of amblyopia is ranged between 1.3 to $3.3 \%$. The highest prevalence of amblyopia is in Canada (4.7\%), while the lowest prevalence of amblyopia was in Singapore (1.19\%) [2-6]. This variation of prevalence among different countries could be due to different vision screening programs used for pre-school children, which plays a major role in detecting different visual problems. Hence, early treatment of the condition and preventing visual complications [2]. 
The conventional treatment of Amblyopia is patching the good eye or penalization. Although patching treatment has a significant role in improving the visual acuity in amblyopic patients, it has some disadvantages including recurrence of amblyopia, lack of commitment and the visual acuity in the amblyopic eye may not reach to normal visual standard (i.e. $6 / 6$ in some cases) [7].

Binocular treatment is a new approach that is recommended to treat amblyopia such as playing binocular I Pad games with wearing red-blue goggles to preserve eye binocularity. Previous studies provided evidence about the efficacy of binocular I Pad treatment in improving the visual functions in amblyopic eye than patching [7, 8]. However, no one compares its outcome. Thus, the purpose of this study was to differentiate between two different binocular I Pad games in improving visual functions of amblyopic eyes in preschool children.

\section{Method}

\subsection{Participants Recruitment}

This study was approved by the ethics committee of deanship of scientific research at King Saud University. The protocol of the study was explained to each participant at the time of recruitment and informed consent was obtained according to the Declaration of Helsinki.

Children with untreated amblyopia of both genders aged from 5 to 9 years were enrolled in this pilot study. Amblyopia was defined as "best-corrected visual acuity $\geq 0.2 \log$ MAR and an intraocular difference of $\geq 0.2 \log$ MAR associated with the presence or history of strabismus, anisometropia, or both in an otherwise healthy child" [9].

Children with amblyopia were recruited from Pediatric ophthalmology clinic of a tertiary care hospital and were divided into three groups based on treatment: Group $1=8$ children treated with patching only, Group $2=5$ children attributed to play binocular snake I Pad game with patching, and Group $3=5$ children attributed to play binocular blocks I Pad game with patching. Children were classified based on the type and severity of amblyopia i.e. strabismic, anisometropic or combined.

\subsection{Procedure}

Visual acuity VA, refraction, stereoacuity, and ocular alignment tests were performed for all participants in the first visit and 4 weeks after starting the treatment. Visual Acuity was measured using Lea symbol chart, Stereo acuity was tested using titmus fly test and Refraction was tested by cyclo retinoscopy. Ocular alignment test was measured in primary position at near fixation using cover uncover and alternative cover test. Visual functions were compared between both visits.

\subsection{Binocular I Pad Games}

Two games were selected in this study: snake game (figure 1) and blocks game (figure 2). Although these two games have similar mechanism in the treatment of amblyopia, they differ in the playing criteria. Snake game is a pursuit game, while blocks game is building game. The child was asked to wear red-blue goggles added to his optical correction for half an hour while playing the binocular I Pad games. These goggles present the high-contrast images to the amblyopic eye, while the low-contrast image was presented to the normal fellow eye. The blue filter placed over the amblyopic eye to provide the amblyopic eye with contrast of $100 \%$, while the red filter placed over the fellow eye to provide contrast of $20 \%$ [9, 10]. Compliance form was designed including the time and duration of patching and playing I pad game. This form was given to parents to record treatment compliance.

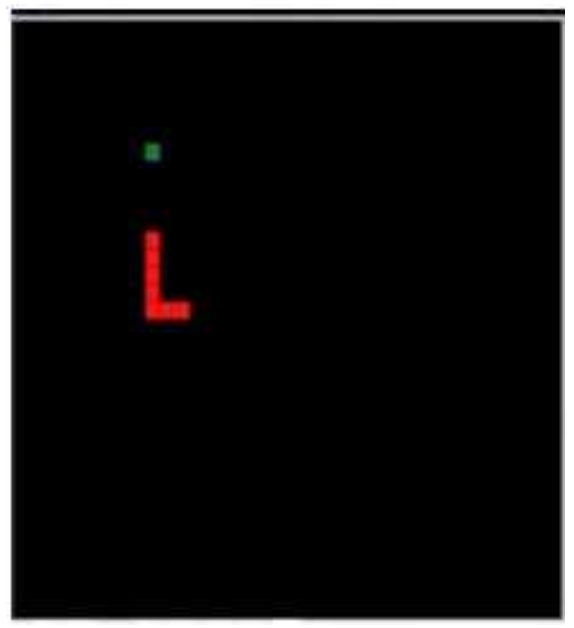

Figure 1. Binocular snake IPad game.

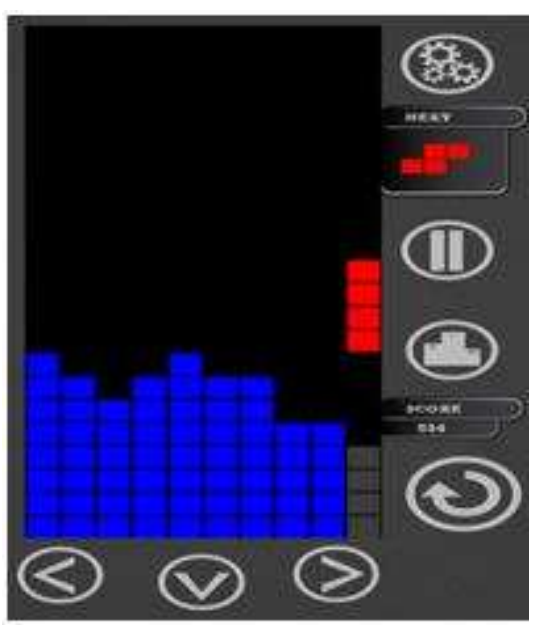

Figure 2. Binocular blocks game.

\subsection{Statistical Analysis}

Statistical analysis was performed using SPSS version 22.0. All variables were expressed as Mean \pm Standard deviation. The paired T-test was used to evaluate the difference between measurements of each group. The confidence interval was set at $95 \%$ and probability values of $\mathrm{P}<0.05$ were considered statistically significant. 


\section{Result}

A total of 18 children of both genders aged from 5 to 9 years with untreated amblyopia were enrolled in this pilot study. There was no significant difference in gender ( $P$ value $=0.7)$, and age $(P$ value $=0.3)$ between the three groups . The characteristics of studied groups are shown in table 1 .

Table 1. The characteristics of studied groups.

\begin{tabular}{llll}
\hline & Patching only N=8 & Snake game +patching $\mathbf{N}=\mathbf{5}$ & Blocks game +patching N=5 \\
\hline Age $($ mean \pm SD) & $4.8 \pm 1.72$ & $6.4 \pm 2.4$ & $6.4 \pm 8.1$ \\
Gender M (\%) & $5(62 \%)$ & $2(40 \%)$ & $2(50 \%)$ \\
Type of amblyopia N (\%) & & & \\
Anisometropic & $4(50 \%)$ & $1(20 \%)$ & $2(50 \%)$ \\
Strabismic & $2(25 \%)$ & $3(60 \%)$ & - \\
Combined & $2(25 \%)$ & $1(20 \%)$ & $2(50 \%)$ \\
VA in amblyopic eye (mean $\pm \mathrm{SD})$ & $0.48 \pm 0.25$ & $0.4 \pm 0.9$ & $0.6 \pm 0.11$ \\
VA in normal eye (mean $\pm \mathrm{SD})$ & $0.19 \pm 0.22$ & $0.12 \pm 0.07$ & $0.3 \pm 0.16$ \\
Degree of amblyopia N (\%) & & & - \\
Mild & $4(50 \%)$ & $2(40 \%)$ & $5(100 \%)$ \\
Moderate & $3(37.5 \%)$ & $3(60 \%)$ & - \\
Severe & $1(12.5 \%)$ & - & $3552 \pm 0$ \\
Stereopsis $(\operatorname{mean} \pm \mathrm{SD})$ & $3552 \pm 0$ & $3552 \pm 0$ & \\
\hline
\end{tabular}

Follow up visit

After four weeks, ten children with amblyopia visited the clinic for follow up $(n=4$ Amblyopic children who underwent patching treatment only, $n=2$ children played binocular snake game in addition to patching, $n=3$ children played blocks I Pad game in addition to patching). Table 2 demonstrates the visual functions of participants.

Table 2. The visual functions of children attending their follow up visit after 4 weeks.

\begin{tabular}{|c|c|c|c|c|c|c|c|c|c|}
\hline \multicolumn{3}{|c|}{ Patching only $\mathrm{N}=4$} & \multicolumn{3}{|c|}{ Snake game+patching $N=2$} & \multicolumn{4}{|c|}{ Patching only $N=4$} \\
\hline & $1^{\text {st }}$ & $2^{\text {nd }}$ & \multirow{2}{*}{ P value } & \multirow{2}{*}{$\begin{array}{l}1^{\text {st }} \\
\text { Visit }\end{array}$} & \multirow{2}{*}{$\begin{array}{l}2^{\text {nd }} \\
\text { Visit }\end{array}$} & \multirow{2}{*}{ P value } & \multirow{2}{*}{$\begin{array}{l}1^{\text {st }} \\
\text { Visit }\end{array}$} & \multirow{2}{*}{$\begin{array}{l}2^{\text {nd }} \\
\text { Visit }\end{array}$} & \multirow{2}{*}{$P$ value } \\
\hline & Visit & Visit & & & & & & & \\
\hline VA & $0.48 \pm 0.25$ & $0.31 \pm 0.2$ & 0.2 & $0.4 \pm 0.9$ & $0.18 \pm 0.5$ & 0.07 & $0.6 \pm 0.11$ & $0.18 \pm 0$ & 0.01 \\
\hline Stereoacuity & $3552 \pm 0$ & $1967 \pm 181$ & 0.1 & $3552 \pm 0$ & $175 \pm 106$ & 0.005 & $3552 \pm 0$ & $53 \pm 41$ & 0.000 \\
\hline
\end{tabular}

$\mathrm{P}$ value for $\mathrm{VA}<0.01$

$P$ value for steroacuity $<0.05$

Visual acuity was improved in all amblyopic children in the form of one line improvement in patching group, two lines with binocular snake I Pad game and three lines with binocular blocks I Pad game. Moreover, stereoacuity was enhanced in all groups with greatest advance in blocks games group followed by snake group then patching group. The VA and stereoacuity of children who played blocks game was better than the VA of amblyopic children who played snake game ( $p=0.01 \& 0.000$ respectively). Children showed good compliance to the specific treatment given including both patching treatment and I pad games except one child who is excluded from the study.

\section{Discussion}

This current study compared the visual functions (i.e. visual acuity and stereoacuity) in three groups of children with amblyopia who were treated with patching only and two different binocular I Pad games (snake and blocks games). Our data attested that binocular I Pad games augment the visual function of amblyopic eye. Previous studies confirmed that VA and stereopsis were improved in school age amblyopic children who played blocks I Pad game [8, 13] and snake game [11]. On the other hand, other studies showed no improvement $[1,7]$. This is explained by the fact that school aged amblyopic children in these studies were asked to play blocks games only without patching. This suggests that patching the sound eye is a necessity in amblyopia treatment and binocular games are the reason behind the variation in visual functions between these studies.

The present findings proclaimed a greater improvement in stereo acuity, in blocks game than in snake game. Some studies support the present results [8, 13]. However others reported no improvement for most subjects $[1,7]$. This can be assigned to differences in stereo acuity tests used. Binocular games improve the visual functions of amblyopic eye and fasten the treatment of amblyopia as it reduces suppression and increase binocularity.

Children playing snake I Pad game reported that they lost their interest in continuing playing the game after three days of starting the treatment. While children who played blocks game did not have any complaints. This could be due to the different pattern of the two games as in snake game, the child was asked to keep the eye on following different located dotes in attempt to grab them, while in blocks game, the child was asked to fall blocks in order to build over them and create different forms of buildings, which could be more diverse and interesting for children in this age. This could interpret the reason of better 
improvement in VA and stereo acuity in blocks groups relative to snake group.

\section{Conclusion}

In conclusion, this study demonstrated great effectiveness of both blocks, and snake binocular I Pad games on visual functions enhancement for treatment of amblyopia. Furthermore, these games can contribute with patching in achieving more VA and stereo acuity improvement in a shorter duration than patching alone. Although we had a small sample size and treatment remained only 4 weeks, binocular I pad games showed a good potential for treating amblyopia.

\section{Acknowledgements}

This research project was supported by a grant from the research center of the Centre for Female Scientific and Medical Colleges at King Saud University. The authors thank the Deanship of Scientific Research and RSSU at King Saud University for their technical support

\section{Author Contributions}

Kholoud Ahmed Bokhary: Main investigator

Latifah Saud Aldohayan: Ophthalmic examination and data collection

Fahmy Rania Medhat: Writing and scientific revision of the manusript

Abdullah Ghazi Alotaibi: Ophthalmic examination

\section{Author Disclosure Statement}

The authors declare no potential conflicts of interest with respect to the authorship, and/or publication of this article

\section{References}

[1] Friendly DS. Amblyopia: definition, classification, diagnosis, and management considerations for pediatricians, family physicians, and general practitioners. Pediatric clinics of North America 1987; 34: 1389-1401.
[2] Aldebasi YH. Prevalence of amblyopia in primary school children in Qassim province, Kingdom of Saudi Arabia. Middle East African journal of ophthalmology 2015; 22: 8691.

[3] Chia A, Dirani M, Chan YH et al. Prevalence of amblyopia and strabismus in young singaporean chinese children. Investigative ophthalmology \& visual science 2010; 51: 34113417.

[4] Doheny Eye Institute, Department Of Ophthalmology. Prevalence of amblyopia and strabismus in African American and Hispanic children ages 6 to 72 months the multi-ethnic pediatric eye disease study 2008; 115: 1229-1236.

[5] Muhammad A A, Imran A, Asad AK. Prevalence of Amblyopia among Government Middle School Children in City Of Lahore, Pakistan. International Journal for Agro Veterinary and Medical Sciences 2010; 4: 41-46.

[6] Drover JR, Kean PG, Courage ML et al. Prevalence of amblyopia and other vision disorders in young Newfoundland and Labrador children. Canadian journal of ophthalmology Journal canadien d'ophtalmologie 2008; 43: 89-94.

[7] Menon V, Shailesh G, Sharma P et al. Clinical trial of patching versus atropine penalization for the treatment of anisometropic amblyopia in older children. Journal of the official publication of the American Association for Pediatric Ophthalmology and Strabismus J AAPOS 2008; 12: 493-97.

[8] Holmes JM, Beck RW, Kraker RT et al. Impact of patching and atropine treatment on the child and family in the amblyopia treatment study. Arch ophthalmol 2003; 121: 1625-32.

[9] Birch EE, Li SL, Jost RM et al. Binocular iPad treatment for amblyopia in preschool children. Journal of the official publication of the American Association for Pediatric Ophthalmology and Strabismus J AAPOS 2015; 19: 6-11.

[10] Ali Rastegarpour. A computer-based anaglyphic system for the treatment of amblyopia. Clin ophthalmo 2011; 23: 131923.

[11] Von Noorden GK, Campos EC. Binocular Vision and Ocular Motility: Theory and Management of Strabismus 2002. London: Mosby.

[12] Manh VM, Holmes JM, Lazar EL, Kraker RT et al. A Randomized Trial of a Binocular iPad Game Versus Part-Time Patching in Children Aged 13 to 16 Years with Amblyopia. Am J Ophthalmol 2018; 186: 104-115.

[13] Ferri FF. Ferri's Clinical Advisor. 2017. Philadelphia: Elsevier. First edition. 